\title{
The Integrated Hazard Analysis Integrator
}

\author{
A. Terry Morris* \\ NASA Langley Research Center, Hampton, Virginia 23681 \\ Michael J. Massie ${ }^{\dagger}$ \\ ARES Corporation, Houston, Texas 77058
}

\begin{abstract}
Hazard analysis addresses hazards that arise in the design, development, manufacturing, construction, facilities, transportation, operations and disposal activities associated with hardware, software, maintenance, operations and environments. An integrated hazard is an event or condition that is caused by or controlled by multiple systems, elements, or subsystems. Integrated hazard analysis (IHA) is especially daunting and ambitious for large, complex systems such as NASA's Constellation program which incorporates program, systems and element components that impact others (International Space Station, public, International Partners, etc.). An appropriate IHA should identify all hazards, causes, controls and verifications used to mitigate the risk of catastrophic loss of crew, vehicle and/or mission. Unfortunately, in the current age of increased technology dependence, there is the tendency to sometimes overlook the necessary and sufficient qualifications of the integrator, that is, the person/team that identifies the parts, analyzes the architectural structure, aligns the analysis with the program plan and then communicates/coordinates with large and small components, each contributing necessary hardware, software and/or information to prevent catastrophic loss. As viewed from both Challenger and Columbia accidents, lack of appropriate communication, management errors and lack of resources dedicated to safety were cited as major contributors to these fatalities. From the accident reports, it would appear that the organizational impact of managers, integrators and safety personnel contributes more significantly to mission success and mission failure than purely technological components. If this is so, then organizations who sincerely desire mission success must put as much effort in selecting managers and integrators as they do when designing the hardware, writing the software code and analyzing competitive proposals. This paper will discuss the necessary and sufficient requirements of one of the significant contributors to mission success, the IHA integrator. Discussions will be provided to describe both the mindset required as well as deleterious assumptions/behaviors to avoid when integrating within a large scale system.
\end{abstract}

\section{Nomenclature}

CIS $=$ conditional independence statement

$\mathrm{CPD}=$ conditional probability distribution

DAG = directed acyclic graph

DRM = design reference mission

ESMD $=$ Exploration Systems Mission Directorate

FHA $=$ Functional Hazard Analysis

$\boldsymbol{G} \quad=$ a graph of topological dependence structure

$I(., . \mid$.) $\quad=$ a conditional independence rule

ISS $\quad=$ International Space Station

IHA $=$ Integrated Hazard Analysis

JPD $\quad=$ joint probability distribution

NASA $=$ National Aeronautics and Space Administration

\footnotetext{
* Safety-Critical Avionics Systems Branch, Mail Stop 130, AIAA Lifetime Associate Fellow.

† Lead for NASA's Constellation Integrated Hazard Analysis, ARES Corporation, Johnson Space Center.
} 


$\begin{array}{ll}P(), p() & =\text { probability distribution } \\ p\left(x \mid \pi_{x}\right) & =\text { a conditional probability distribution } \\ \pi_{x} & =\text { parents of node } x \\ \text { SAVIO } & =\text { Software and Avionics Integration Office } \\ S E \& I & =\text { Systems Engineering \& Integration } \\ \boldsymbol{X}, \boldsymbol{Y} & =\text { sets of variables } \\ x, y & =\text { instantiated variables }\end{array}$

\section{Introduction}

Rapid globalization and pervasive use of new technology are having profound effects on the systems we work with and the tasks we do. Today's leaders, managers and integrators must adapt quickly from old patterns of behavior in order to be effective in today's complex systems environment ${ }^{1}$. For example, history has shown the National Aeronautics and Space Administration (NASA) that previous spaceflight successes are no guarantee for future spaceflight success given the 1986 Challenger and 2003 Columbia Space shuttle disasters. As an initial gut response to failure, spacecraft engineers and flight managers may initially want to focus their efforts on building better hardware or acquiring more reliable software technologies that enable redundant fault tolerant mechanisms or produce more robust algorithms to aid decision making processes in order to reduce or prevent future crew/vehicle/mission loss. Despite these initial gut reactions, engineers and managers would benefit greatly by analyzing the true systemic contributors to many accidents, namely, the people, their interactions, and the environments (organizational culture, values, mindsets and processes) in which they operate. As viewed from both Challenger and Columbia accidents, lack of appropriate communication, management errors and lack of resources dedicated to safety were cited as major contributors to both fatalities ${ }^{6}$. The lesson here states that despite the pervasive use of new technology, the type of people selected and employed to produce the end product are just as, or more, important to mission success than mere efforts used to design the hardware, write the software or devise operational procedures. The purpose of this paper is to discuss the critical nature of the integrator (a type of leader, manager, etc.) within a complex system and to reveal mental and emotional attributes (mindsets) required to achieve mission success in today's evolving organizational climate.

In order to elucidate various dimensions of this topic, this paper will provide background information on the special nature of complex systems, particularly NASA's Constellation program, as well as explain the evolutionary trends that have occurred to produce today's complex systems and the leaders required to operate within them. An analysis of the IHA integrator will be provided from both external and internal perspectives to reveal essential human capabilities to strategically lead and tactically manage within the IHA environment. Additionally, discussions will be provided to identify necessary or sufficient requirements that a present-day integrator must embody to be effective in today's complex systems environment. Finally, the paper will describe a qualitative emotional competence dependency model that can be used by human resource departments in a quantitative evaluation used for hiring.

\section{Background}

To fully explain current requirements of the IHA integrator, background will be provided on the evolution of organizations, present day complex systems, NASA's Constellation system-of-systems architecture (as one type of complex system), leaders and managers, the function of IHA and IHA integrators, and the subsequent evolution of the integrator (one type of leader, manager, etc.) as a response to present day complex systems.

\section{A. The Evolution of Organizations}

Ever since the 1960's, large organizations have been evolving in order to compete in an ever changing marketplace and global economy. Fundamental infrastructure and philosophical shifts have occurred during this time that have forced organizations to evolve, to die, or to become greatly diminished in their effectiveness. The current business landscape embodies sweeping changes that include accelerating globalization, massive amounts of information and a dearth of leaders with ethics and integrity willing to place the good of the organization above personal advantage. It is important to note that these sweeping changes have affected and redefined the underlying nature of organizations and the leaders that operate within them. Albert Vicere, Professor of Strategic Leadership at 


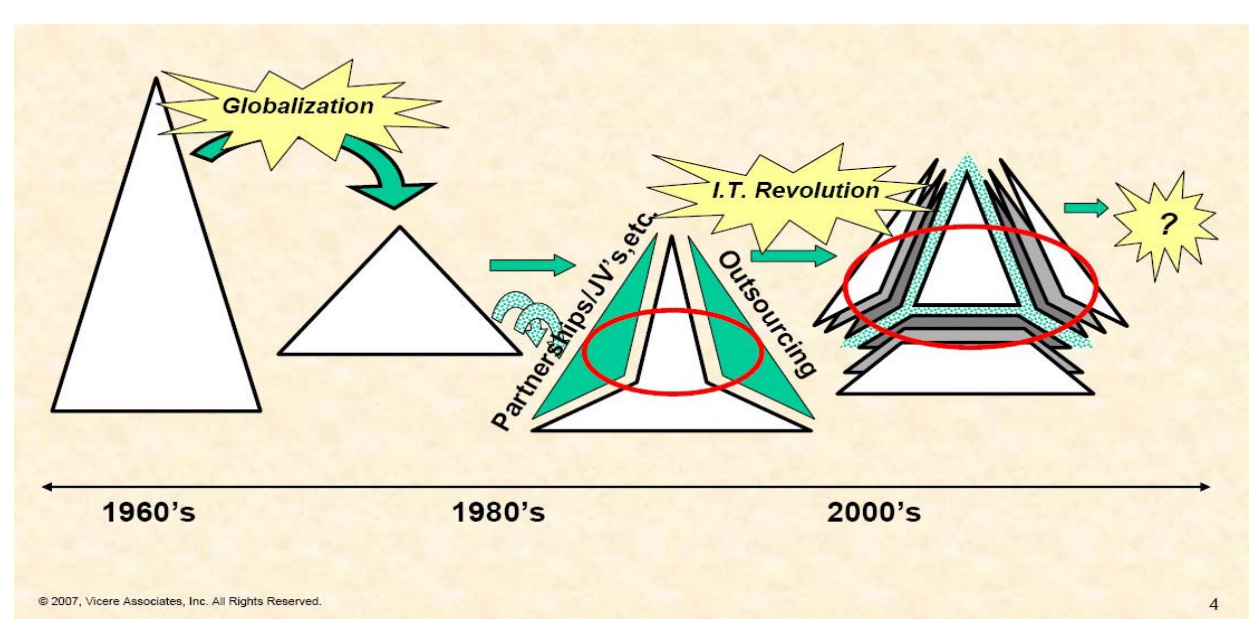

Figure 1. Evolving Organizational Forms ${ }^{7,9}$

Pennsylvania State University's Smeal College of Business Administration, clearly articulates the two major inflection points ${ }^{7}$ that have driven the emergence of the current "networked economy." These inflection points are globalization and information technology.

As depicted in Professor Vicere's evolving organizational forms (figure 1), older organizations in the 1960's were built on the notion of control, that is, controlled structure, controlled hierarchy and controlled resources (this is represented by the leftmost large triangle). When the first major inflection point, globalization, began to challenge US companies, these large companies could not compete with the faster, more nimble global competitors. In order to compete, large US companies evolved to organizational models that were flatter and faster, hence the smaller, flatter triangle in figure 1. Another major change that followed globalization was the concept of "core competencies" developed and implemented by large organizations in the 1990's. "Core competencies" aided many companies by focusing their organization on those functions that they wanted to do better than anyone else ${ }^{8}$. As more companies bought into the core competency framework, they had to learn to lose a little more control and to subsequently allow others to supply operational tasks that were previously done in-house. These tasks generally fell outside of their core competencies. This led to an evolved organizational structure that permitted outsourcing arrangements, joint ventures and tactical partnerships ${ }^{7}$.

The second major inflection point, outlined by Professor Vicere, was the information technology revolution. This revolution now enabled companies to work in strategic and tactical partnerships linked by powerful information networks ${ }^{7}$. The infusion of information technology in the workplace (telecommunication networks, e-commerce solutions, web-conferencing, etc.) allowed organizations to streamline their businesses in new and creative ways. These information technology capabilities allow for tightly coupled partnerships that fundamentally changed the nature of organizations. These newer organizational structures require network-enabled employees, that is, employees that are required to network through strategic partnerships via information technology infrastructures to fulfill organizational obligations. This organizational structure is represented by the shadow pyramid, the single triangle surrounded by multiple teams and partnerships (rightmost in figure 1). Employees not only need to master their own competency but they must also be able to simultaneously network with multiple sources, organizations, partnerships, or teams in order to deliver on organizational commitments.

The older organizational forms had processes primarily based on control, while the newer, more networked organizations rely on processes based on relationships. The distinction between the two cannot be overstated. Employees can take their relationships with them to other organizations if the control infrastructure is too confining or too lax. Some leaders who were effective in older organizational structures are generally not very effective in today's networked environment. Besides the changed organizational nature from one of control to one of relationships, there also exists the need for leaders to function productively and effectively with another aspect of current organizations, the uncomfortable nature of today's complex systems.

\section{B. Present Day Complex Systems}

As expressed very succinctly by Yaneer Bar-Yam at the New England Complex Systems Institute, "the traditional approach to large engineering projects follows the paradigm established by the Manhattan project and the Space program ${ }^{10}$." This paradigm is embodied in four underlying assumptions. First, projects generally want to 


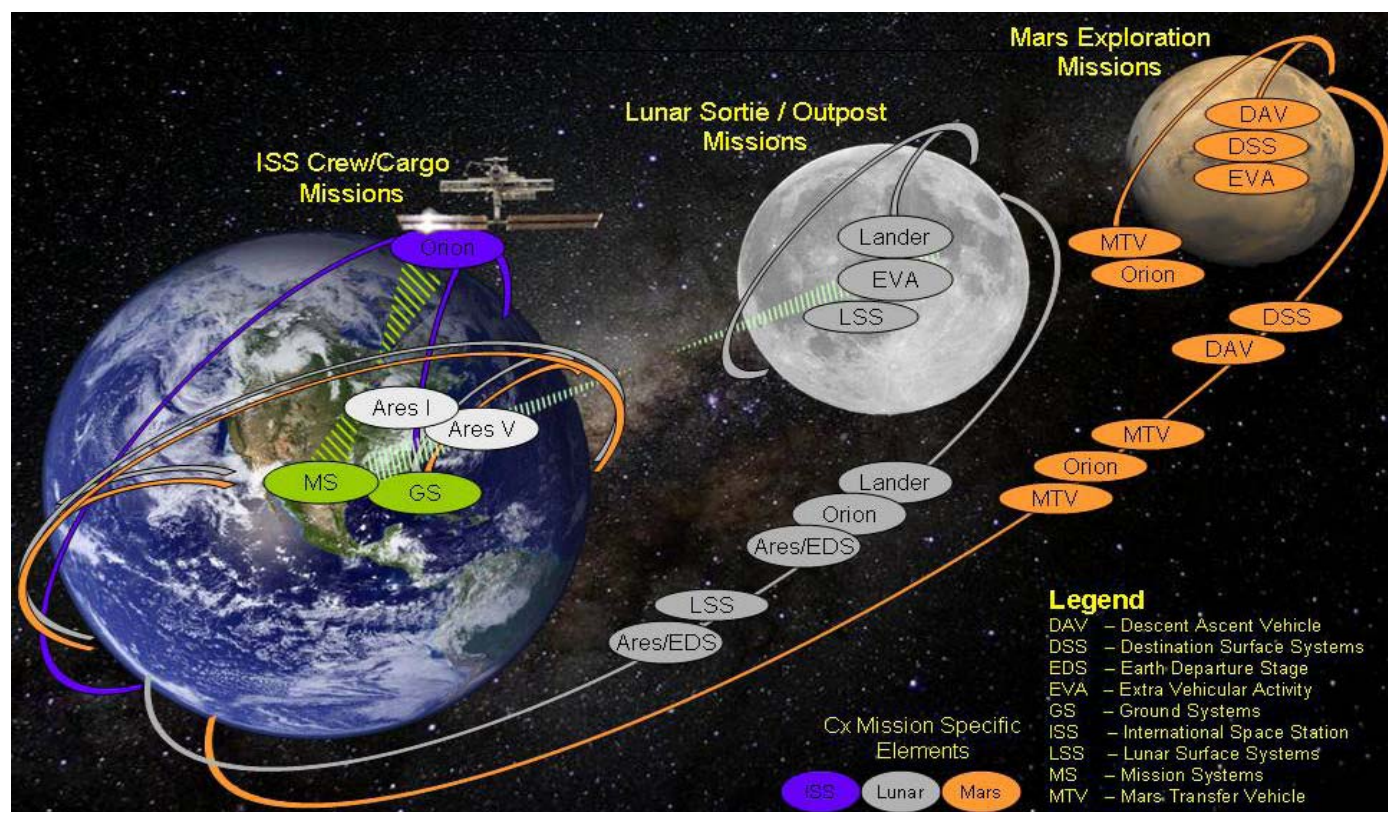

Figure 2. Constellation Architecture Concept ${ }^{14}$

replace old obsolete systems with new systems by using new technology. Second, the new technology is based upon a clear understanding of the basic principles or equations that govern the system. Third, the goal of the project and its more specific objectives are clearly understood. And fourth, a design will be developed and implemented that will subsequently lead to accomplishing the mission. These underlying assumptions generally accompany organizational efforts to build large engineering projects. Many organizations have even adopted formal system engineering processes as a way of methodically moving through each phase of a large project. However, several present-day organizations, even the ones employing formal system engineering processes, have failed to achieve their goals or objectives. Many of these failures are due to a misunderstanding and misapplication of system engineering processes. System engineering, like all other tools, is appropriate for certain types of problems. Standard system engineering techniques tend to fail when the inherent complexity of the goal, objectives or functions is beyond some threshold. Inherent complexity below this threshold appears to allow for the application of standard system engineering processes. Inherent complexity at or above this threshold appears to require the use of complex system engineering approaches ${ }^{11}$ or system-of-systems engineering techniques ${ }^{12}$. This implies that insight and discernment are difficult when dealing with complex systems.

One of the underlying reasons for this difficulty can be expressed by the concept of local spatiotemporal causality $^{13}$. Local spatiotemporal causality is a condition that is amenable to human reasoning, that is, humans find it easy to understand causality when the effect is local or close to the cause in either space (distance) or time. Conversely, humans have extreme difficulty perceiving or understanding causality when there is distance or a large amount of time between the cause and the eventual effect. From an aerospace hazard perspective, this difficultly can be seen when a satellite launch system experiences lightning on the launch pad, but the subsequent malfunction to some lower level avionics box is not manifest until after the satellite has separated from the launch system, has correctly reached stationary orbit and has operating successfully for several days. The causal linkage is not readily apparent to most people due to the distance between the cause (lightning on the pad) and the effect (a malfunctioning avionics box) in both space and time.

This difficulty in connecting the causal dots is just one significant difference between standard and complex systems. Other differences include the ability to understand indirect and highly nonlinear processes, designing systems that permit heteroscedastic control, etc.

\section{NASA Constellation's System of Systems Architecture}

On January 14, 2004, the President directed NASA to embark on a robust space exploration program that will advance the nation's scientific, security, and economic interests. In support of this vision the United States will:

a. Implement a sustained and affordable human and robotic program to explore the solar system and beyond. 
b. Extend human presence across the solar system, starting with a human return to the Moon by the year 2020, in preparation for human exploration of Mars and other destinations.

c. Develop the innovative technologies, knowledge, and infrastructures both to explore and to support decisions about future destinations for human exploration.

d. Promote international and commercial participation in exploration to further US scientific, security, and economic interests.

In response to the US Space Exploration Policy, NASA established the Exploration Systems Mission Directorate (ESMD) to develop a constellation of new capabilities, supporting technologies, and foundational research that allow for the sustained and affordable exploration of space. The Constellation Program was subsequently established within ESMD to oversee the human exploration mission development program. In addition, Congress enacted the NASA Authorization Act of 2005, establishing that the Agency "shall establish a program to develop a sustained human presence on the Moon, including a robust precursor program, to promote exploration, science, commerce, and United States preeminence in space, and as a steppingstone to future exploration of Mars and other destinations ${ }^{14} . "$

The Constellation Program, one example of a present-day complex system, is a tightly coupled program that consists of multiple projects that develop, manage, and execute portions of the Constellation human exploration mission. No single project is capable of implementing a complete mission. The design reference missions (DRM) fall into three general categories: missions to the International Space Station (ISS), missions to the moon, and missions to Mars (figure 2). The ISS missions consist of ISS crew rotation missions and cargo delivery missions. The lunar missions provide the capability to transport and land humans on the Moon, operate for a limited period on the surface, and safely return them to Earth. Lunar missions also allow for exploration of high-interest science sites or scouting of future lunar outpost locations. The Mars missions provide the capability to transport and land humans on Mars, operate for a limited period on the surface, and safely return them to Earth ${ }^{14}$.

There are many management and integration tasks that need to be completed successfully for each Constellation mission. Some of these include managing, coordinating and synchronizing multiple schedules, addressing individual and systemic risks, incorporating the insertion of new technology into the technical baseline, developing/tailoring the system-level architecture, and performing integrated hazard analyses.

\section{Leaders and Managers in Present Day Organizations}

As present-day organizations continue to evolve, they tend to require both leaders and managers that work well within the cultural or organizational contexts of the time. What is the difference between leaders and managers in present-day organizations? According to Warren Bennis and Dan Goldsmith ${ }^{26}$, "A good manager does things right. A leader does the right things." The essence of their argument has led to the generally-accepted consensus that management involves power by position whereas leadership involves power by influence. Despite the consensus, there exist differences of opinion between the function of leader and the function of manager. According to Michael Maccoby's book "The Leaders We Need", "There is only one irrefutable definition of a leader, and that is someone people follow....Leadership always implies a relationship between leader and led, and that relationship exists within a context. Leaders who gain followers in one context...may not attract followers in a different context...To understand leadership in context, we have to place ourselves within that culture and get inside the heads of the people a would-be leader is trying to mobilize." Maccoby continues by stating, "In the global marketplace of our time, the leadership context has changed from that of fifty years ago, when corporate bureaucracies rode along in stable, predictable markets. Then, managers were needed to plot a steady course; innovative leaders could be seen as disruptive and were often sidelined. Now, in the context of continual change brought about by knowledge workers, leadership is needed not only at the top, but also throughout companies. Furthermore, different types of leaders are needed to integrate projects and teams of technical professionals working across department and national borders: strategic (leaders who communicate a vision with a compelling sense or purpose), operational (leaders who build the organization and infuse the energy that transforms visions into results) and network or bridge-building (leaders who facilitate the understanding and trust that turns different types of specialists into collaborators). These leadership roles are most effectively filled by different types of people in terms of their intellectual skills and personalities. Furthermore, these leaders need to understand each other in order to work together ${ }^{1}$."

Generally, the analysis in this paper focuses on one type of leader within the organization, the systems integrator. The integrator can be seen (from Maccoby's perspective) as a bridge-building type of leader who at times need strategic, operational and management capabilities to adequately perform the job. Using NASA's Constellation program as one example of a complex system, the authors have tended to infer, throughout the paper, characteristics that are true for leaders and managers in general to specific traits found in integrators who perform integrated hazard 
analyses. To be clear, many of the inferences made in the paper are intended to be general and not succinct statements of fact. Further investigation or analysis is needed to ascertain which specific leadership traits or results can be definitively mapped to the IHA role.

\section{E. The Function of Integrated Hazard Analysis and the IHA Assessor}

One of the primary functions that must occur across any highly complex endeavor is one aspect of the safety analysis process, namely, the development of an informative, beneficial and insightful integrated hazard analysis. The goal of IHA is to provide insight into risks and hazards so that program and project managers can mitigate possible hazardous behavior from ground equipment, launch assets, space vehicles, astronaut suits and their various interactions before catastrophes occur. Hazards, from this perspective, incorporate all conditions that lead to loss of crew, loss of vehicle and loss of mission. The IHA process for Constellation, for example, performed at the architecture and system level also includes hazards that impact the International Space Station, the public and international partners. The iterative IHA process involves documenting inter-system hazards and their causes, ensuring that the need for controls necessary for safe integrated operation are properly addressed and documented in system or element hazard reports and analyzing the integrated system to ensure coverage and completeness. The depth of the IHA is commensurate with the level of detail provided to the program as it matures through each major milestone.

Michael Massie, former contractor Safety Manager for development of the IHA on the International Space Station responsible for hazard identification and mitigation and present lead for Constellation's IHA, has described four essential keys to success that all integrators must understand in order to achieve safe results and to prevent from being overwhelmed by the enormity of the IHA task ${ }^{15}$. These four essential elements are 1) define the analysis structure, 2) provide a good IHA plan, 3) provide for good and reliable communications and 4) select and utilize the right personnel for the job. These elements provide a solid foundation for tackling the hardware, software, operations, information flow, team member constituency, politics and their varied interactions that characterize present-day complex systems.

\section{F. The Necessity of Integrator Evolution}

Regardless of the requirements levied on integrators in the past, present-day integrators must be able to operate in today's evolved organizational structures (ones based primarily on relationships without absolute control), be able to discern and apply appropriate tools commensurate with the level of inherent complexity of the task, be able to grasp and recognize local spatiotemporal causality (and know how to mitigate its effects), be able to understand large scale architectures, its objectives and challenges, and be able to communicate and work with differing mindsets at various levels of an organization in order to accomplish the difficult task of integration. With changes in the organizational environment of complex systems, particularly NASA Constellation program, there exists the need for integrator personnel to evolve in order to handle the additional and myriad tasks associated with operating within today's complex systems.

\section{Analyzing the Integrated Hazard Analysis Integrator}

If the organizational impact of leaders, managers and safety personnel contribute more significantly ${ }^{5}$ to mission success than purely technological components (hardware, software, operations, etc.), then what competencies or capabilities should managers seeking to fill personnel positions look for when evaluating these individuals ${ }^{16}$. In order to analyze required capabilities, we will focus on the role of the system integrator (one type of leader, manager, etc.) within the context and function of integrated hazard analysis. Keep in mind that the analysis described in this paper, though focused on the integrator role, is applicable to various roles ${ }^{16}$ that have similar or comparable functionality within large scale system contexts. These roles include requirements owners, system designers, system analysts, validation and verification engineers, logistics and operations managers, customer interface personnel, technical managers, information managers, process engineers and system coordinators.

The analysis in this paper will uncover personnel behaviors and characteristics from the reports that analyzed both Challenger and Columbia accidents from organizational perspectives ${ }^{5,6}$. The analysis will proceed by digging deeper into the psychological, affective and volitional functions of the human brain (using national/international experts in comparative human functioning and executive leadership) to extract necessary and sufficient conditions that generally characterize leaders and managers that are needed in our present-day complex systems environments. As stated earlier, general inferences have been made from leader and manager characteristics to specific traits 


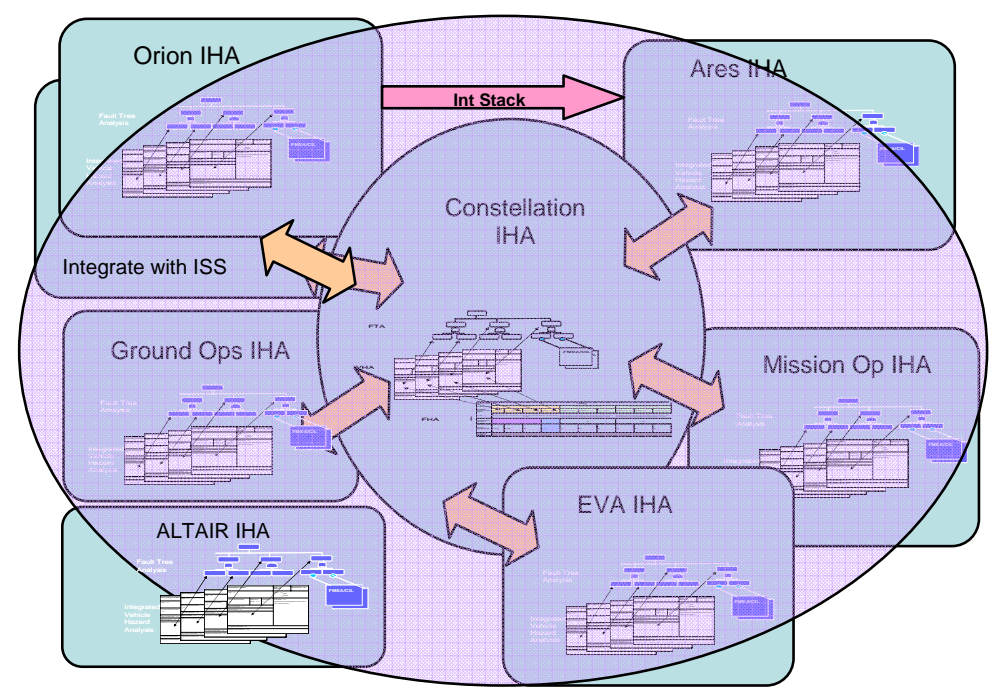

Figure 3. Constellation's Integrated Hazard Analysis Structure

required by integrators who perform integrated hazard analyses. IHA integrators, from this perspective, are seen as a type of network or bridge-building leader and manager. The analysis will briefly describe the mindsets and behaviors that should be avoided when managers or integrators are working within a large system-of-systems. The analysis will conclude with a description of a present day leader that satisfies necessary and sufficient conditions for an IHA integrator.

\section{A. What is required by an Integrator?}

As discussed earlier, Massie has prescribed the four keys to success that if adopted will lead the integrator of hazards to accomplish the enormous task of integrating various systems within a complicated system. These keys reveal strategic, operational and organizational lessons learned from previous IHA experiences. Once again, the four keys are 1) define the analysis structure, 2) provide a good IHA plan, 3) provide for good and reliable communications and 4) select and utilize the right personnel for the job. Despite the brevity of Massie's approach to success, there exists a dynamic operational environment that each integrator will experience differently based on personality traits, inborn characteristics, mental biases, expertise and personal disposition. Though no two integrators are alike, there exists a common set of diverse tasks that must be balanced, performed and cultivated as the IHA end product matures. In short, integration, if done correctly, is hard work.

On any given day, the function of an IHA integration involves understanding the major parts of the overall system (see figure 3 for Constellation IHA), being cognizant of the political, social and environmental factors that influence the organizational culture, and having a good grasp on the technical challenges as you influence and lead the team toward coherent and cohesive integration. Current day integrators need to acknowledge the importance of relationships both individually and collectively. Note that the shadow pyramid structure in figure 1 is very similar to Constellation's IHA structure shown in figure 3. This does not appear to be a coincidence. This similarity reveals the importance of one-on-one and collective relationships in order to accomplish integration goals. At times, the integrator will have to develop a flexible structure in order to accommodate the insertion of divergent hazard analyses. At other times, the integrator will need to be the advocate and the educator, the unyielding boss and the accommodating friend, the benefactor and the scapegoat. Integrators need to proclaim their vision with conviction while remaining open to criticism. While complying with programmatic requirements, the integrator must also know when to push back, particularly when an organization has established self-defeating practices that prohibit safety or is moving on a path that leaves the integration goal unattainable. And while the integrator is speaking truth to power or presenting results to an independent safety panel or upper management board, he/she must also be cognizant of the mental models of others, putting forth much effort not to offend unnecessarily and to protect, when appropriate, the psychological makeup of superiors or stakeholders who are in error.

This last note cannot be overemphasized. Sometimes the boss, the customer or the stakeholder is wrong. Sometimes they do not know what they really want. John Gibson, an early pioneer of systems engineering at the University of Virginia, wrote a decalog ${ }^{17}$ where he revealed that "the customer (usually) does not understand the 
problem." In these common cases, it is the integrator's responsibility to work with the customer to help him gain a proper understanding of the problem and to select the best solution path. Unfortunately, this is not usually easy.

\section{B. What Was Missing in the Past (Challenger and Columbia)?}

Nancy Leveson at the Massachusetts Institute of Technology has investigated technical and managerial factors that led to both space shuttle Challenger and Columbia losses $^{5}$. Her research posits that almost identical management and organizational factors were identified in both the Rogers Commission ${ }^{18}$ for Challenger and the Columbia Accident Investigation Board report ${ }^{19}$ for Columbia. Accidents, from her perspective, are better viewed as complex processes involving direct and indirect interactions between people, organizational structures, engineering activities and physical systems ${ }^{6}$. The following conditions, in her analysis, are some of the preconditions that led to the disasters: performance pressures and program survival fears gradually led to an erosion of rigorous processes; a decrease in system safety program priority led to budget cuts in the safety activities that eventually led to a situation of (unrecognized) high risk where an accident became almost inevitable; huge barriers against dissenting opinions by stating preconceived conclusions based on subjective knowledge and experience, rather than solid data; reliability engineering was substituted for safety; the safety information system was ineffective; and hazard analysis, when it was performed, was not adequate.

Given these results, what can be done when making selections of integrators of IHA systems who will promote safety while preventing or mitigating these negative conditions? From these findings, it can be deduced that integrators should be selected who maintain rigorous processes to ensure safety. Additionally, integrators should also establish an environment where dissenting options are encouraged and opinions are backed-up with solid data. Integrators are also expected to use appropriate tools for the task at hand as well as protect the infrastructure system containing the data by which critical decisions are made. The type of individual that succeeds here is one who learns from past mistakes (their own and others), is open for critical feedback, whose ethics and integrity are foundational and who strives to improve the communication paths in the organizational both vertically and horizontally.

\section{Digging Deeper - Ingredients of Superb Job Performance: IQ, Expertise and Emotional Intelligence}

In the previous sections, we analyzed requirements for an IHA integrator from external perspectives, that is, from day-to-day functions that any integrator must complete, from strategic, operational and organizational perspectives and from analyses of accident reports that uncovered required duties that promote safety. In this section, we will endeavor to dig deeper by analyzing the integrator from an internal perspective, that is, required functionality from psychological, affective and volitional dimensions. The goal of this section is to analyze the ingredients of superior job performance for humans that operate in technical, leadership, managerial and organizational capacities very similar to what is required when performing IHA integration within a large systemsof-systems environment.

In order to have common vocabulary and understanding, we will provide a general overview of human brain anatomy and will briefly describe functions associated with specific compartments of the brain. The human brain, the most complex organ in any creature on earth, is actually a set of brains working in concert to achieve daily functioning. During early development of the central nervous system, the three main portions of the brain are the forebrain (the prosencephalon - the forward most portion), the midbrain (the mesencephalon) and the hindbrain (the rhombencephalon). When matured, the forebrain separates into the diencephalon (containing the prethalamus, thalamus, hypothalamus, subthalamus, epithalamus and pretectum) and the telencephalon or cerebrum (containing the cerebral cortex and the basal ganglia). Most of the size of the human brain comes from the cerebral cortex, a convoluted layer of neural tissue that covers the surface of the forebrain. The cerebral cortex (and various compartments like the neocortex) is categorized into sections that perform certain functions (see figure 4 (a)): the frontal lobe - conscious reasoning, making judgments, tempers emotional response, assigns meanings to words; the parietal lobe - sensation, perception, integration of sensory information, manipulation of objects; the temporal lobe - hearing ability, language; occipital lobe - vision ${ }^{20}$. As compared to other mammals, humans tend to have expanded frontal lobes which are involved in executive functions such as self-control, planning, reasoning, and abstract thought. In humans, the cerebrum surrounds older parts of the brain such as the midbrain. The limbic system (see figure 4 (b)) is a complex set of structures that lie on both sides and underneath the thalamus, just under the cerebrum. It includes the hypothalamus, the hippocampus, the amygdala, among other areas. The limbic system is also the source of our fight versus flight reaction. It appears to be primarily responsible for our emotional life, and has a lot to do with the formation of memories. Limbic, olfactory, and motor systems project fibers from the cerebrum to the brainstem and spinal cord. Cognitive and volitive systems project fibers from the cerebrum to the thalamus and to specific regions of the midbrain. The neural connections between the cerebrum and the midbrain facilitate complex behaviors such as social interactions, learning, working memory, speech and language. 


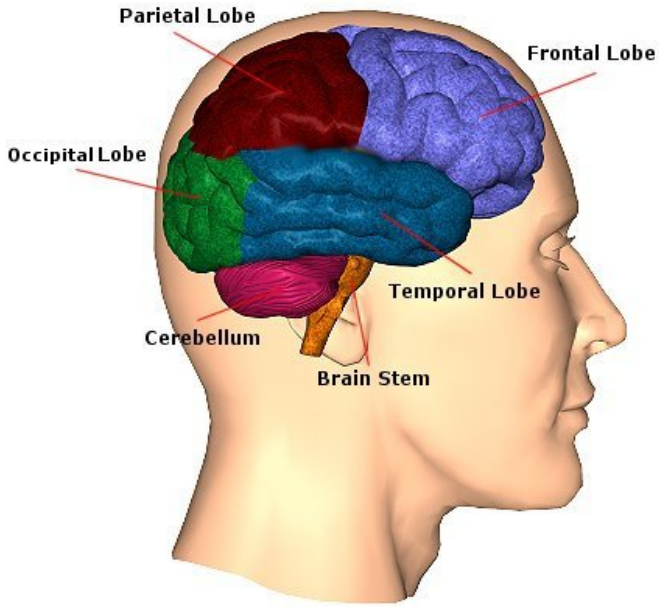

(A) The Cerebral Cortex ${ }^{20}$ (Head - Thought)

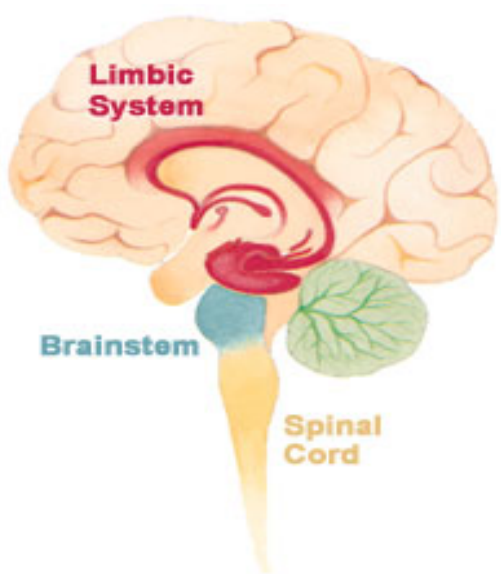

(B) The Limbic System ${ }^{21}$ (Heart-Emotions)

Figure 4. The Human Brain: Head Versus Heart

The reason for describing functional areas of the brain is to underscore two central areas of primary concern in this analysis - the cerebral cortex and the limbic system. The cerebral cortex, particularly the frontal lobe, is seen as the area that allows for conscious thought and helps in making decisions. In olden days, this area has been called "the head." In like manner, the limbic system is seen as the seat of emotions, responsible for emotional life. Because of its proximity to the brain stem and spinal cord, it has a much more immediate impact on the physical human heart that pumps blood throughout the body. In olden days, people would mistake the human heart as the primary area of emotions and passions not realizing that the heart was only responding to neural signals it received from various areas of the brain, primarily midbrain (the limbic system) and the hindbrain (brainstem). In this analysis, we will characterize the limbic system as "the heart," the true seat of emotions. With this basic understanding, we will endeavor to analyze the human brain and its interactions with the environment using national/international experts in comparative human functioning and executive leadership to extract necessary and sufficient conditions that characterize integrators (a type of leader, manager, etc.) that are needed in our present-day complex systems environments.

\section{Necessary but not Sufficient Requirements: Cognitive Ability (IQ)}

Cognitive abilities are brain-based skills and mental processes that are needed to carry out tasks. Cognitive abilities are believed to function like muscles, that is, if they are not used regularly, your cognitive abilities will atrophy over time. In the past, standardized tests were developed to measure how much intelligence was embodied by each person as a way of determining top performers in industry and government. The intelligence quotient or IQ was the standard by which intelligence and superior job performance was measured for a number of decades even though it has had dissenters that argued its validity. IQ, in general, is associated with the cerebral cortex (figure 4 (a)), particularly the prefrontal cortex ${ }^{4}$, but was believed to be something unchangeable in the human brain. In the 1950's and 1960's, personality tests in addition to IQ were seen as major ingredients for job excellence. Current research has overturned much of the thinking of IQ as the primary means of identifying top performers. Some studies have shown that IQ contributes at most 20 percent to the factors that determine life success ${ }^{22}$. Brain development studies at the National Institute of Mental Health have recently suggested that experience and environmental cues may play a very important role in shaping intelligence ${ }^{23}$. Daniel Goleman, a pioneer in analyzing emotional intelligence, has provided much insight explaining the limits of IQ. In his book, Working With Emotional Intelligence ${ }^{4}$, he explains how IQ alone at best leaves 75 percent of job success unexplained. He continues by stating that IQ, paradoxically, has the least power in predicting success among people smart enough to handle the most cognitively demanding fields like engineering, law or medicine. From Goleman's perspective, IQ "is just a threshold competence - you need it to get in the field, but it does not make you a star." Competence, in this analysis, is a personal trait or set of traits that leads to more effective or superior job performance. These results appear to have a clear mapping to the integration function. When performing the integration function, the IHA 
integrator needs to have the cognitive ability to organize the IHA plan, to reason between competing structural approaches, to analyze the interactions between the various systems and to perform trade-offs for decision making under uncertainty. Because IQ is a threshold competence, cognitive ability is a necessary requirement for an IHA integrator. However, it is not a sufficient requirement when viewed from a singular competence perspective.

\section{Necessary but not Sufficient Requirements: Expertise}

Practical intelligence is a combination of technical know-how and experience. Expertise, in this light, is a combination of common sense plus specialized knowledge or skill that is picked up while doing a job. It incorporates the tricks of the trade that can only be learned while in the trenches ${ }^{4}$. In relation to IQ, Robert Sternberg, an authority on success and intelligence, revealed that expertise contributes at least as much as IQ when it comes to success on the job ${ }^{24}$. As expressed in Goleman's research, "practical intelligence is rarely the main factor in outstanding job performance." Expertise, therefore, is a baseline competence. You need it to get the job done. Managers and supervisors of technical workers need to have some degree of expertise in order to reasonably understand what their employees are doing. However, when it comes to superior performers, expertise is a threshold requirement because the primary ability demonstrated by outstanding managers and integrators is not technical, but rather related to handling people ${ }^{4}$. When performing the integration function, the IHA integrator needs to have a certain depth of knowledge about many technical areas that are generally only gained after years in the integration role. Thus, expertise is a necessary requirement for an IHA integrator. However, it is not a sufficient requirement when viewed from a singular competence perspective because other factors such as interaction with people are needed.

\section{Necessary but not Sufficient Requirements: Emotional Intelligence}

In Goleman's research, emotional intelligence is simply the capacity for recognizing our own feelings and those of others, for motivating ourselves, and for managing emotions well in ourselves and in our relationships ${ }^{4}$. Though distinct from academic intelligence, it is best viewed as complementary to cognitive ability as measured by IQ. The relative importance of emotional intelligence to IQ is clearly seen in the workplace. Many book smart people who lack emotional intelligence end up working for people who have lower IQ's but excel in emotional intelligence abilities. This statement is not intended as a judgment, but only as a general statement of what is required when interaction with numerous people is an integral part of the integration task. Intellect and emotions represent two different kinds of intelligence that express activity of different parts of the brain. The intellect is based solely on the workings of the neocortex (part of the cerebral cortex involved in higher functions such as sensory perception, generation of motor commands, spatial reasoning, conscious thought and language), the evolved layers at the top of the brain, while the emotional centers are lower in the brain (the limbic system). Emotional competence involves the emotional centers (the limbic system) at work in concert with the intellectual centers ${ }^{4}$.

For clarification, there is a difference between emotional intelligence and emotional competence. Emotional intelligence determines our potential for learning practical skills that are based on five elements: self-awareness, motivation, self-regulation, empathy and social skills ${ }^{4}$. Emotional competence expresses how much of that potential we have translated into on-the-job capabilities. Just because a person is high in emotional intelligence does not guarantee the person will have learned the emotional competencies that matter for work.

The significance of emotional intelligence over IQ and expertise in determining superior job performance is clear and convincing. Goleman's research clearly shows that far more weight is given to emotional competence than to cognitive ability. Even among scientists and those in technical professions, analytical thinking ranks third after the ability to influence and the drive to achieve ${ }^{4}$. Influencing and achieving are components of emotional competence. Analysis and independent results confirm the following: emotional competence was found to be twice as important in contributing to excellence on-the-job compared to pure intellect and expertise ${ }^{4}$. In the US government, Goleman revealed that the higher the level of the job, the less important technical skills and cognitive ability were, and the more important competence in emotional intelligence became.

With respect to job complexity, Goleman described research that revealed the following results: for simple jobs, those in the top one percent (in terms of top performers) produced three times more output than those in the bottom one percent; for jobs of medium complexity, a top performer was twelve times more productive than those at the bottom; for the most complex jobs, performers in the top one percent produced 127 percent more than average performers. In summary, these research results show that for those involved with high complexity jobs, like IHA integration, emotional intelligence is not simply additive with cognitive ability, it is multiplicative. Emotional intelligence is clearly the predominant ingredient in star performance on the job. 
Given the relationship between emotional intelligence and emotional competence, it is clear that emotional intelligence does not guarantee a person will develop and display associated emotional competencies. Additional factors such as organizational climate and the person's interest in the job also play a part to determine whether the competence will manifest itself. When performing the integration function, the IHA integrator needs to have emotional intelligence in order to reasonably select and utilize the right personnel for tasks, to increase communication and understanding between team members and to resolve personnel and organizational disputes. Despite the significance of emotional intelligence over IQ and expertise for complex jobs such as IHA, emotional intelligence is only a necessary requirement. It is not a sufficient requirement when viewed from a singular competence perspective because how the emotional intelligence is manifested in the integrator role is a major ingredient (among others) that is needed.

\section{Necessary but not Sufficient Requirements: Big Picture Thinking (Systems Perspectives)}

Though Goleman more than adequately revealed the relative importance of emotional intelligence over IQ and expertise, his research also distinguished the most significant cognitive ability with respect to star performers in executive and leadership positions. This cognitive ability was "big-picture" thinking. It is a form of pattern recognition that allows leaders to pick out meaningful trends from the deluge of surrounding information allowing them to think strategically far into the future ${ }^{4}$. With respect to high-performing CEOs, three clusters of competencies were found that distinguished them from average CEOs. The first two competencies were emotional while the third was cognitive, that is, strategic, conceptual, big-picture thinking. It was noticed that the greatest leaders did something that even the top performing leaders did not. The greatest leaders integrated their emotional realities into their inspiring visions allowing them to instill strategy with meaning. Even though Goleman's research focus was on executive and leadership positions like CEOs and the IHA integrator role as described in this paper is a somewhat different type of leadership role, the authors believe that many (not all) of the results described are applicable to the IHA integrator. This is because the iterative nature of the IHA process involves a tumultuous amount of information that must be mined, refined and sifted. The ability to extract meaningful patterns from the deluge of data presented is a critical function found in only the best integrators. The best integrators, like the high-performing CEO's, tend to incorporate the emotional realities of the organizational culture into their strategic analysis of mitigating hazards.

Howard Gardner, the influential Harvard theorist, aptly described the cognitions of big-picture thinking in his book, Five Minds for the Future ${ }^{3}$. In this book, Gardner describes "the synthesizing mind" as the ability to take information from disparate sources and to integrate ideas from different disciplines into a coherent whole in ways that make sense. This capacity, according to Gardner, will become more crucial as information continues to mount at dizzying rates.

Michael Massie, present lead for Constellation's IHA, also described this "big-picture” thinking capability when he discussed the type of person it takes to operate within the IHA environment. In his words, "It takes a special kind of thinker to wrap their head around a large scale integrated hazard analysis. Those who succeed can simultaneously see many parts working together on many levels. The person, for example, who can see the quarks joined to become electrons which fly around in a cloud around the nucleus of the atom which is joined to other atoms to form a molecule which is added to other molecules to from a mixture of matter which is joined with more matter to form a planet which orbits a sun with moons encircling it and the sun is circumnavigating a galaxy which is part of a cluster which is part of a supercluster which is part of the cosmos. The person who can see this and track it in his/her mind and freely shift from layer to layer is the individual who can handle the IHA of large scale integrated systems ${ }^{15}$." Though big-picture thinking is significant among top performers, it is a necessary but not sufficient requirement for an IHA integrator.

\section{Deleterious Mindsets, Assumptions and Behaviors to Avoid}

In the process of performing IHA integration, there are many mindsets, assumptions and behaviors that will impede the integrator's ability to safely and efficiently perform the job. Goleman, who already discussed the importance of emotional intelligence over IQ and expertise, also identifies behaviors that caused leaders to fail. (If we continue to describe the integrator as a type of leader or manager, then many of Goleman's leadership results can be applied to the IHA integrator.) Two significant behaviors include rigidity and poor relationships. Rigidity is defined as the integrators inability to adapt their style to changes in the organizational culture. Poor relationships begin by being too harshly critical, insensitive or demanding. Other deleterious behaviors include work overload, lack of autonomy and unfairness. 
Tavris and Aronson in their lively book, titled, Mistakes Were Made (but not by me) ${ }^{2}$, describes the condition of cognitive dissonance. In their own words, "The brain is designed with blind spots, optical and psychological, and one of its cleverest tricks is to confer on us the comforting delusion that we, personally, do not have any. In a sense, dissonance theory is a theory of blind spots - of how and why people unintentionally blind themselves so that they fail to notice vital events and information that might make them question their behavior or their convictions ${ }^{2}$." Because most of us are not self-correcting, we should endeavor to keep telling ourselves the truth, even when we make major mistakes. The IHA integrator will undoubtedly encounter blind spots in his/herself as well as blind spots in others. The dynamics of cognitive dissonance is relevant when a team member/stakeholder is obviously wrong in their assessment, but they choose self-justification of their beliefs instead of humility to the truth.

\section{E. Putting it All Together (Necessary and Sufficient): Interactive Collaborators}

Michael Maccoby, in his book "The Leaders We Need", tackled the subject of leaders and followers throughout time to reveal insightful characteristics of past and present-day leaders. His research parallels the organizational shifts through time described earlier by Professor Vicere (see figure 1). He analyzed the psychological makeup of leaders from old organizational control paradigms and from present-day "knowledge worker" organizations. He calls those stuck in old organizational behaviors "Bureaucratic Followers." He also contrasts these individuals with a new type of leader called "Interactive Collaborators." To be sure, there are some individuals who successfully transition from the former role to the latter. In his analysis, the Interactive Collaborator is less focused on status and autonomy (compared to the Bureaucratic Follower) and more focused on teamwork and self-development. Their strengths include their independence, the readiness for change and their quick ability to connect with others and work in a self-managed team. They tend not to follow autocratic, insensitive bosses who don't listen. They are natural communicators and collaborators. They tend to be aware of their own transferences and generally rely on others (usually an outsider) to provide reality checks. In short, the Interactive Collaborator combines multiple capacities (emotional, strategic, big-picture thinking, etc.) into a cohesive whole in order to provide leadership to organizations. For this reason, Interactive Collaborators meet the condition of necessary and sufficient requirements for an IHA integrator.

\section{Emotional Competence Dependency Model}

Goleman's groundbreaking research has revealed the additive importance of emotional intelligence relative to IQ and expertise for regular jobs as well as its multiplicative importance when applied to highly complex jobs. If emotional competence is the real world application of emotional intelligence, then how can managers and human resource departments use Goleman's emotional intelligence-to-competence relationships when assessing the emotional competencies of potential candidates in the hiring process. One suggestion is to use Goleman's expert knowledge in emotional intelligence and emotional competence to develop a formal, graphical, qualitative dependency model that can be used in a quantitative evaluation. There would be many advantages to such a model. First, it could be adjusted to several types of employer contexts, that is, weights could be applied to each of the five dimensions of emotional intelligence (self-awareness, self-regulation, motivation, empathy and social skills) to fit the emotional requirements of the employer. Second, given the type of inference employed, the model can be used to handle uncertainty associated with partial or missing data which generally occurs in hiring situations. Third, the graphical representation could be used to justify the reasoning thereby clearly explaining why a candidate is hired or not in a consistent and unambiguous fashion. Dr. Terry Morris, a NASA researcher with expertise in commercial off-the-shelf (COTS) software evaluations, developed a process by which expert knowledge could be extracted and used formally in a comparative evaluation ${ }^{25}$. This section will be used to describe how Morris's process can be adapted in developing an emotional competence dependency model.

Dependency is a statement about a set of variables. Saying that variables $\boldsymbol{A}$ and $\boldsymbol{B}$ are dependent on each other means that if the value of $\mathbf{A}$ is known, then information has been provided (to some extent) about the value of variable $\boldsymbol{B}$. Dependency can be conditional or unconditional, unidirectional or bidirectional, and functional or causal. The type of dependency of interest in this analysis is causal pair wise conditional dependency. Dependency models, as used in this analysis, are sets of dependency statements. Formally, a dependency model is a pair $\boldsymbol{M}=(\boldsymbol{U}$, $I$ ), where $\boldsymbol{U}$ is a finite set of elements or variables, and $I(., . \mid$.) is a rule that assigns truth values to a three place predicate whose arguments are disjoint subsets of $\boldsymbol{U}$. The interpretation of the conditional independence assertion $I(\boldsymbol{X}, \boldsymbol{Y} \mid \boldsymbol{Z})$ is that having observed $\boldsymbol{Z}$, no additional information about $\boldsymbol{X}$ could be obtained by also observing $\boldsymbol{Y}$. In a probabilistic model, $I(\boldsymbol{X}, \boldsymbol{Y} \mid \mathbf{Z})$ holds if and only if 


$$
\boldsymbol{P}(\boldsymbol{x} \mid \mathbf{z}, \boldsymbol{y})=\boldsymbol{P}(\boldsymbol{x} \mid \mathbf{z}) \text { whenever } \boldsymbol{P}(\mathbf{z} \mid \boldsymbol{y})>0
$$

for every instantiation $\mathrm{x}, \mathrm{y}$ and $\mathrm{z}$ of the sets of variables $\boldsymbol{X}, \boldsymbol{Y}$ and $\boldsymbol{Z}$.

A graphical representation of a dependency model $\boldsymbol{M}=(\boldsymbol{U}, I)$ is a direct correspondence between the elements in $\boldsymbol{U}$ and the set of nodes in a given graph, $\boldsymbol{G}$, such that the topology of $\boldsymbol{G}$ reflects the independence assertions of $I$. There are different kinds of graphical models. The most common are undirected graphs (Markov networks) and directed graphs (Bayesian networks). Each one has its own merits and shortcomings. These graphical models are knowledge representation tools used by an increasing number of scientists and researchers. The reason for the extended success of graphical models is their capacity to represent complexity and to handle independence relationships, which has proved crucial for the storage of information. The graphical models used in this paper will be restricted to Bayesian networks.

Graphical models that represent directed dependencies are known as Bayesian networks and they result in a powerful knowledge representation formalism based on probability theory. Bayesian networks are graphical models where the nodes represent random variables, the arcs signify the existence of direct causal influences between the variables, and the strengths of these influences are expressed by forward conditional probabilities ${ }^{28}$. Formally, a Bayesian network is a pair, $\mathbf{B}=(\boldsymbol{G}, \boldsymbol{P})$, defined by a set of variables $\boldsymbol{X}=\left(X_{1}, \ldots, X_{n}\right)$, where $\boldsymbol{G}$ is a directed acyclic graph (DAG) defining a model $\boldsymbol{M}$ of conditional dependencies among the elements of $\boldsymbol{X}$,

$$
\boldsymbol{P}=\left(p\left(x_{1} \mid \boldsymbol{\pi}_{1}\right), \ldots, p\left(x_{n} \mid \boldsymbol{\pi}_{\boldsymbol{n}}\right)\right)
$$

is a set of $n$ conditional probability distributions (CPDs), one for each variable, and $\boldsymbol{\pi}_{\boldsymbol{i}}$ is the set of parents of node $\boldsymbol{X}_{\boldsymbol{i}}$ in $\boldsymbol{G}$. The set $\boldsymbol{P}$ encodes the conditional independence assumptions of $\boldsymbol{G}$ to induce a factorization of the joint probability distribution (JPD) as

$$
p(x)=\prod_{i=1}^{n} p\left(x_{i} \mid \pi_{i}\right) .
$$

When the random variables are discreet, as used in this analysis, the types of distribution applied to each variable will be multinomial, thereby describing a multinomial Bayesian network. In multinomial Bayesian networks, all variables in $\boldsymbol{X}$ are discrete, that is, each variable has a finite set of possible values. An advantage of Bayesian networks is its natural perception of causal influences thus making it an unambiguous representation of dependency. This is useful for the emotional competence evaluation problem in that it allows for the explicit identification of influences between attributes of each potential candidate. Moreover, the Bayesian network's requirement of strict positivity allows it to serve as an inference instrument for logical and functional dependencies. Furthermore, its ability to quantify the influences with local, conceptually meaningful parameters allows it to serve as a globally consistent knowledge base. In this way, Bayesian networks are natural tools for dealing with uncertainty and complexity. Characterizations of these networks generally involve determining whether the structure of the model is known and whether the data is complete. Bayesian models (when the structure is unknown) may be constructed from prior knowledge, conditional independence statements (CIS), data, or some hybrid of the above. Prior knowledge may include distinct knowledge of a problem domain as well as input from experts. As used in this analysis, Bayesian networks, $\mathbf{B}=(\boldsymbol{G}, \boldsymbol{P})$, are graphical and mathematical models that represent the dependency and probabilistic structure between a set of variables. They can be used intuitively as a knowledge base for a set of variables where $\boldsymbol{G}$ (dependency structure) is the structural component and $\boldsymbol{P}$ (the probabilistic structure) is the more quantitative component. The dependency structure $(\boldsymbol{G})$ of the emotional competence Bayesian network will be extracted from domain expert, Daniel Goleman. The term probability model refers to a complete specification of the JPD over a set of variables. Therefore, the terms probability model and JPD are used interchangeably. The JPD contains structural as well as quantitative information about the relationships among the variables. We will use the term dependency model to refer only to the causal structure of the relationships among a set of variables. The emotional competence dependency model extraction process described here will be a modified version of the one Morris used for COTS software evaluation. This extraction process will describe the transformation procedure that maps the emotional competence dependency structure to a graphical representation of that structure as a dependency model.

The extraction process starts by identifying emotional intelligence and emotional competence relational/causal phrases that define expert-revealed relationships. Causal relations can generally be identified by scanning for the following words: 'affects', 'influences', ‘is crucial for', ‘contributes to', etc. Generally, when no causal relation can 
be identified, the attribute/variable/node is considered independent. The following phrases were identified in Goleman's book, Working With Emotional Intelligence ${ }^{4}$ :

1) Note $1 \rightarrow$ there are five dimensions of emotional intelligence (self-awareness, self-regulation, motivation, empathy and social skills)

2) Note $2 \rightarrow$ The emotional competencies cluster into groups, each based on a common underlying emotional intelligence capacity

3) Causal phrase $1 \rightarrow$ self-awareness influences emotional awareness, accurate self-assessment and selfconfidence

4) Causal phrase $2 \rightarrow$ self-regulation influences self-control, trustworthiness, conscientiousness, adaptability and innovation

5) Causal phrase $3 \rightarrow$ motivation influences achievement drive, commitment, initiative and optimism

6) Causal phrase $4 \rightarrow$ empathy influences understanding others, developing others, service orientation, leveraging diversity and political awareness

7) Causal phrase $5 \rightarrow$ social skills influence communication, influence, conflict management, leadership, change catalyst, building bonds, collaboration and cooperation and team capabilities

8) Causal phrase $6 \rightarrow$ self-awareness is crucial for self-regulation and empathy

9) Causal phrase $7 \rightarrow$ self-regulation and self-awareness contribute to motivation

10) Causal phrase $8 \rightarrow$ self-awareness, self-regulation, motivation and empathy are at work in social skills

Where possible, each causal relation was translated into a conditional independence statement and gathered into a causal list. When $\boldsymbol{X}$ and $\boldsymbol{Y}$ are conditionally independent given $\boldsymbol{Z}$, we write $I(\boldsymbol{X}, \boldsymbol{Y} \mid \boldsymbol{Z})$. The statement $I(\boldsymbol{X}, \boldsymbol{Y} \mid \boldsymbol{Z})$ is referred to as a CIS. This statement conveys the idea that once $\boldsymbol{Z}$ is known, knowing $\boldsymbol{Y}$ can no longer influence the probability of $\boldsymbol{X}$. A good way to translate a causal relation to a CIS is by the use of the form

$$
I\left(X_{i}, \boldsymbol{\phi} \mid \boldsymbol{\pi}_{\boldsymbol{i}}\right), \quad i=1, \ldots, n
$$

where $\boldsymbol{X}=\left(X_{1}, \ldots, X_{n}\right)$ represents the set of attribute nodes and $\boldsymbol{\pi}_{\boldsymbol{i}}$ represents the set of parents that influence $\boldsymbol{X}_{\boldsymbol{i}}$. Table 1 displays the CIS for each emotional competence causal phrase described by Goleman. After reconciling any conflicting or missing causal relationships, structural forms of CPDs can be generated and aggregated to form the JPD structure. Each CIS implies a conditional probability distribution of the form

$$
I\left(X_{i}, \boldsymbol{\phi} \mid \pi_{i}\right) \Leftrightarrow p\left(X_{i} \mid \pi_{i}\right) \quad i=1, \ldots, n
$$

The CPD and graphical CPD representation for each CIS in Table 1 is shown in Table 2.

Table 1. Causal List for Emotional Competence Relationships.

\begin{tabular}{|c|l|}
\hline Causal Phrase & CIS \\
\hline $\begin{array}{c}1 \text { (self_aware } \rightarrow \text { em_aware, } \\
\text { acc_self_ass, self_conf) }\end{array}$ & I(em_aware, acc_self_a ss, self_conf, $\phi \mid$ self_aware ) \\
\hline $\begin{array}{c}2 \text { (self_reg } \rightarrow \text { self_ctl, trust, } \\
\text { consc, adapt, innov) }\end{array}$ & I(self_ctl, trust, consc, adapt, innov, $\phi \mid$ self_reg ) \\
\hline $\begin{array}{c}3 \text { (motiv } \rightarrow \text { ach_drive, } \\
\text { commit, init, optim) }\end{array}$ & I(ach_drive, commit, init, optim, $\phi \mid$ motiv ) \\
\hline $\begin{array}{c}\text { 4 (emp } \rightarrow \text { under_oth, } \\
\text { dev_oth, serv_ori, lev_div, } \\
\text { pol_aware) }\end{array}$ & I(under_oth, dev_oth, serv_ori, lev_div, pol_aware, $\phi \mid$ emp ) \\
\hline $\begin{array}{c}\text { 5 (soc_skills } \rightarrow \text { comm, infl, } \\
\text { con_mgmt, lead, ch_cat, bb, } \\
\text { cc, team_cap) }\end{array}$ & I(comm, infl, con_mgmt, lead, ch_cat, bb, cc, team_cap, $\phi \mid$ soc_skills ) \\
\hline $\begin{array}{c}\text { 6 (self_aware } \rightarrow \text { self_reg, } \\
\text { emp) }\end{array}$ & I(self_reg, emp, $\phi \mid$ self_aware ) \\
\hline $\begin{array}{c}\text { 7 (self_reg, self_aware } \rightarrow \\
\text { motiv) }\end{array}$ & I(motiv, $\phi \mid$ self_reg, self_aware ) \\
\hline $\begin{array}{c}\text { 8 (self_aware, self_reg, } \\
\text { motiv, emp } \rightarrow \text { soc_skills) }\end{array}$ & I(soc_skills , $\phi \mid$ self_aware, self_reg, motiv, emp ) \\
\hline
\end{tabular}


Table 2. CPDs and Graphical CPD Representations for Emotional Competence Relationships.

\begin{tabular}{|c|c|c|}
\hline $\begin{array}{l}\text { Causal } \\
\text { Phrase }\end{array}$ & $\overline{\mathrm{CPD}}$ & Graphical CPD Representation \\
\hline 1 & $\begin{array}{c}\text { p(em_aware , acc_self_a ss, } \\
\text { self_conf | self_aware ) }\end{array}$ & \\
\hline 2 & $\begin{array}{l}\text { p(self_ctl , trust, consc, } \\
\text { adapt, innov | self_reg) }\end{array}$ & \\
\hline 3 & $\begin{array}{l}\text { p(ach_driv e, commit, } \\
\text { init, optim | motiv) }\end{array}$ & \\
\hline 4 & $\begin{array}{l}\text { p(under_ot h, dev_oth, serv_ori, } \\
\text { lev_div, pol_aware | emp) }\end{array}$ & \\
\hline 5 & $\begin{array}{l}\text { p(comm, infl, con_mgmt, lead, } \\
\text { ch_cat, bb, cc, team_cap | soc_skills ) }\end{array}$ & \\
\hline 6 & p(self_reg, emp | self_aware ) & \\
\hline 7 & $p($ motiv | self_reg, self_aware $)$ & \\
\hline 8 & $\begin{array}{l}\text { p(soc_skil ls | self_aware , self_reg, } \\
\text { motiv, emp) }\end{array}$ & \\
\hline
\end{tabular}




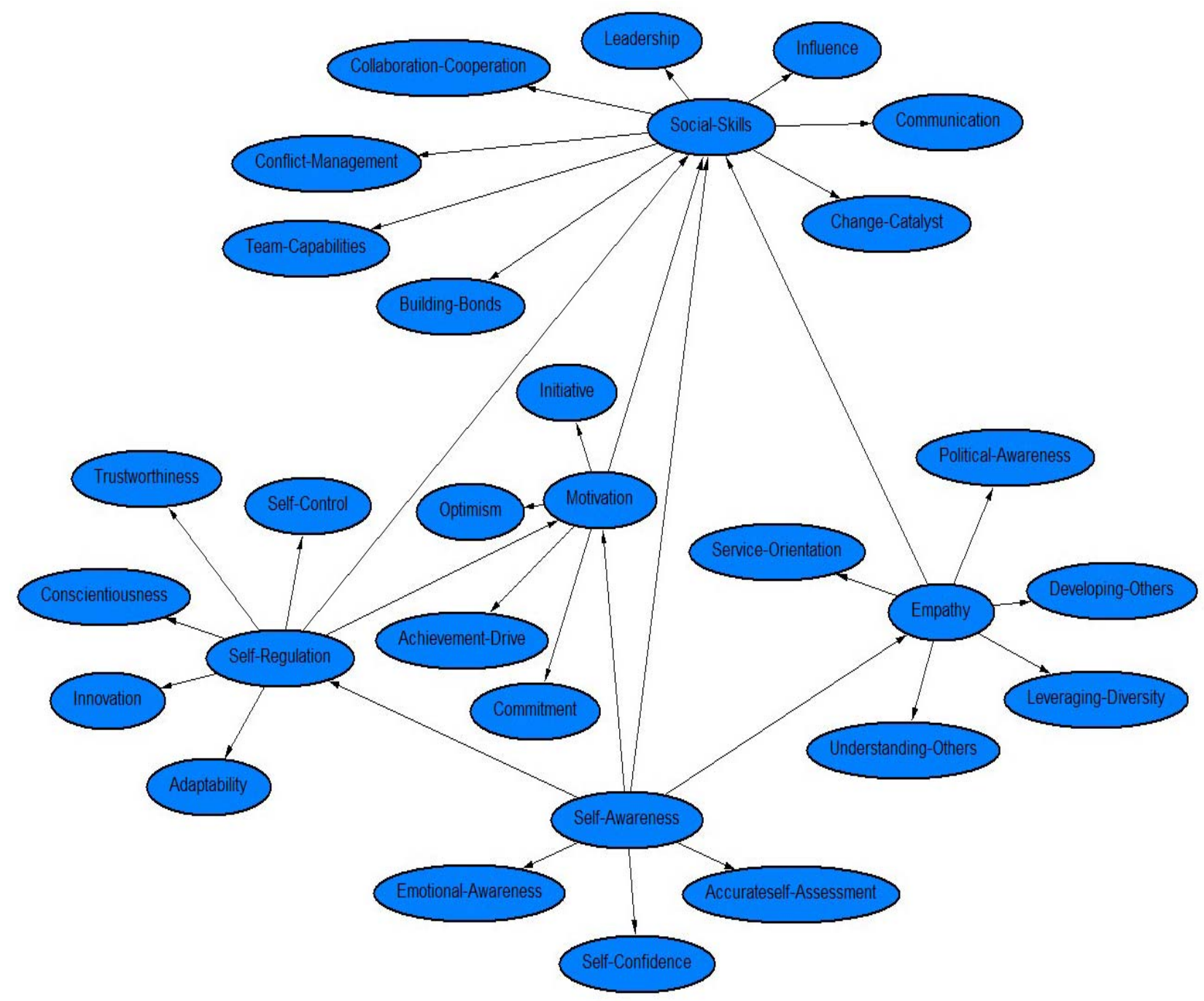

Figure 5. Emotional Competence Dependency Model.

Aggregation of all of the CPDs forms the causal structure of the following joint probability distribution using the factorization in equation (3):

$$
\begin{aligned}
J P D= & p(\text { soc_skills } \mid \text { self_aware }, \text { self_reg, motiv, emp }) \\
& p(\text { self_reg, emp } \mid \text { self_aware }) p(\text { motiv } \mid \text { self_reg, self_aware }) \\
& p(\text { comm, infl, con_mgmt, lead, ch_cat, bb, cc, team_cap | soc_skills }) \\
& p(\text { under_oth, dev_oth, serv_ori, lev_div, pol_aware | emp }) \\
& p(\text { em_aware, acc_self_a ss, self_conf } \mid \text { self_aware }) \\
& p(\text { self_ctl, trust, consc, adapt, innov | self_reg }) \\
& p(\text { ach_drive, commit, init, optim | motiv })
\end{aligned}
$$

Representing the JPD graphically allowing only one node for each emotional competence reveals the emotional competence dependency model in figure 5. This formal model incorporates all the emotional intelligence domain knowledge described by Goleman. Using Bayesian analysis, this dependency model can be tailored and used by managers and human resource personnel in a quantitative evaluation model used for hiring. Weights can be adjusted 
and applied to each emotional intelligence dimension depending on the emotional competence hiring requirements, the organizational culture and the complexity of the job. The model also provides for clear justification as to why a candidate was hired or not and the justification can be viewed graphically providing for clarity and consistency of hiring decisions.

\section{Conclusion}

Organizations are evolving and thus the role and qualifications of integrators must also evolve to reflect the unique nature and characteristics of today's complex systems. IHA integrators must acknowledge the need to evolve since our organizations are moving from hierarchical structures based on control to network-enabled structures based on relationships. External requirements for IHA integrators were extracted from space shuttle accidents reports. Given that organizational factors weigh as much or more to safety and hardware selection or software development, necessary and sufficient requirements were analyzed using various experts in comparative human functioning and leadership. In the process of analyzing internal requirements, that is, from psychological perspectives, a dependency model was revealed that may be beneficial in IHA integrator hiring.

\section{References}

${ }^{1}$ Maccoby, Michael, The Leaders We Need: and What Makes Us Follow, Harvard Business School Press, Publishing, Boston, MA, 2007.

${ }^{2}$ Tavris, Carol, and Aronson, Elliot, Mistakes Were Made (But Not by Me): Why We Justify Foolish Beliefs, Bad Decisions, and Hurtful Acts, Harcourt Publishing Company, Orlando, FL, 2007.

${ }^{3}$ Gardner, Howard, Five Minds for the Future, Harvard Business School Press, Boston, MA, 2006.

${ }^{4}$ Goleman, Daniel, Working with Emotional Intelligence, Bantam Books, New York, NY, 1998.

${ }^{5}$ Leveson, Nancy G. and Cutcher-Gershenfeld, Joel, What System Safety Engineering Can learn from the Columbia Accident, NASA Ames Grant, NAG2-1543, MIT, Cambridge, MA.

${ }^{6}$ Leveson, Nancy G., Technical and Managerial Factors in the NASA Challenger and Columbia Losses: Looking Forward to the Future, MIT, Cambridge, MA.

${ }^{7}$ Vicere, Albert A., “Leadership and the Networked Economy,” Journal of Human Resource Planning, Volume 25, 2002.

${ }^{8}$ Hamel, G. and Prahalad, C. K., Competing for the Future, Harvard Business School Press, Boston, MA, 1994.

${ }^{9}$ Vicere Associates, Inc., Web address: http://www.vicere.com.

${ }^{10}$ Bar-Yam, Y., "When Systems Engineering Fails --- Toward Complex Systems Engineering," IEEE International Conference on Systems, Man and Cybernetics, Volume 2, October 2003.

${ }^{11}$ New England Complex Systems Institute, Web address: http://necsi.edu

${ }^{12}$ System of Systems Engineering Center of Excellence, Web address: http://www.sosece.org

${ }^{13}$ Reuger, A. "Local Theories of Causation and the A Posteriori Identification of the Causal Relation,” Erkenntnis, Volume 48: 25-38, 1998.

${ }^{14}$ CxP 70003, Constellation Program Plan, National Aeronautics and Space Administration, Washington, DC. July 2008.

${ }^{15}$ Massie, M. J., “Constellation Integrated Hazard Analysis - Overcoming the Challenges," Third IAASS Conference, Rome, Italy, October 2008.

${ }^{16}$ Sheard, S. A., "Twelve Systems Engineering Roles," Proceedings of the INCOSE Sixth Annual International Symposium, Boston, MA, 1996.

${ }^{17}$ Gibson, J. E., How to do a Systems Analysis and Systems Analyst Decalog, Ivy, Virginia, July 1991.

${ }^{18}$ Rogers, W. P. (chair), Report of the Presidential Commission on the Space Shuttle Challenger Accident, US Government Accounting Office, Washington, DC., 1986.

${ }^{19}$ Gehman, H. (chair), Columbia Accident Investigation Report, US Government Accounting Office, Washington, DC., August 2003.

${ }^{20}$ Center for Neural Skills; http://www.neuroskills.com/brain.shtml

${ }^{21}$ Teen Drug Abuse; http://teens.drugabuse.gov/mom/mom_opi5.php

${ }^{22}$ Goleman, Daniel, Emotional Intelligence, Bantam Books, New York, NY, 1995.

${ }^{23}$ Vedantam, Shankar, "Brain Development and Intelligence Linked, Study Says,” Washington Post, March 30, 2006.

${ }^{24}$ Wagner, R. K, and Sternberg, R. J., "Practical Intelligence in Real-World Pursuits: The Role of Tacit Knowledge,” Journal of Personality and Social Psychology, Volume 49, 1985.

${ }^{25}$ Morris, A. T., and Beling, P. A., "Extracting Acyclic Dependency Models from Quality Standards for COTS Software Evaluation ,” AIAA 1st Intelligent Systems Technical Conference, Chicago, Illinois, Sep. 20-22, 2004.

${ }^{26}$ Bennis, W. and Goldsmith, D., Learning to Lead, Massachusetts: Persus Book, 1997. 